\title{
Enfermería basada en narrativa, poesía y sociopoética para la gestión emocional y de sentimientos
}

\author{
José Siles González y MCarmen Solano Ruiz \\ Departamento de Enfermería Universidad de Alicante, España. \\ Jose.siles@ua.es | https://orcid.org/0000-0003-3046-639X | Carmen.solano@ua.es | \\ https://orcid.org/0000-0001-8720-8397
}

\begin{abstract}
Resumen: El objetivo de este trabajo es desarrollar una herramienta de reflexión y sentimientos que contribuyan a la gestión de emociones de diferentes comunidades de participantes. Para ello, se ha empleado un grupo de poemas derivados de experiencias clínicas en estudiantes de enfermería como base para la identificación y análisis de emociones y sentimientos. Los resultados obtenidos afirman el reto de los docentes en dotar a los futuros profesionales de la capacidad cognitiva y las destrezas emocionales necesarias para gestionar el aluvión de sentimientos complejos e intensos que emergen durante las interacciones entre personas en diferentes contextos, con el fin de prepararlos para el trabajo emocional inherente a su carga laboral y disminuir el riesgo de sufrir dolencias como el "síndrome de burnout". Podemos concluir que los poemas inspirados en experiencias clínicas, en estudiantes constituyen una herramienta facilitadora para el trabajo emocional y los sentimientos generados durante las prácticas clínicas.
\end{abstract}

Palabras Clave: Enfermaría; Poesia; Sociopoética; Emociones; Sentimientos; Investigación Cualitativa.

\section{Introducción}

El trabajo de enfermería implica un contacto entre personas (cuidador-cuidado) que facilita la generación de emociones y sentimientos muy variados en cuanto a tipología e intensidad. Las emociones son reacciones a situaciones que se pueden dar durante la práctica clínica y constituyen formas de adaptación a las contingencias ambientales y personales que son características del trabajo enfermero. Las emociones, por tanto, no evolucionan como sentimientos conscientes, sino como especializaciones de la conducta y adaptaciones fisiológicas (Darwin, 1998; Vigotsky, 2004) que tienen una repercusión en el rendimiento de los alumnos y profesionales de enfermería.

Los principales actos de expresión de los seres humanos y animales no son aprendidos, sino que son innatos o heredados. Las emociones son universales y se expresan de forma verbal y no verbal (las mismas independientemente de las variaciones culturales y geográficas). Tanto las emociones como los sentimientos deben valorarse para considerar su incidencia en la alteración de la atención, la motivación e incluso el estrés, por lo que tienen una gran importancia en el rendimiento del alumno y/o el profesional. Mientras las emociones tienen una rápida respuesta conductual, dado que funcionan como catalizadores del entorno propiciando impulsos favorables o desfavorables hacia ciertas situaciones, personas, acciones, ideas, etcétera, los sentimientos se construyen como consecuencia de la evolución de una emoción (o grupo asociado de ellas), son más estables y forman parte de la conciencia de la persona.

Este capítulo presenta el proyecto de una herramienta reflexiva que contribuye a la gestión de las emociones. 


\subsection{Construcción del perfil del profesional de enfermería}

El rol de la educación superior en la construcción del perfil profesional de enfermería tradicionalmente se ha centrado en la técnica, soslayando el carácter instrumental de la misma y obviando el sujeto esencial de la disciplina como núcleo central de investigación; es decir, el paradigma predominante en la enfermería en general y en la investigación de la disciplina en particular, ha sido el neopositivista (racional-tecnológico), en el que el profesional desempeña tareas de administración de técnicas de forma estandarizada mientras que el paciente se limita a un rol dependiente, pasivo y subordinado a la autoridad del técnico (Siles, 2018a). Asimismo, la metodología predominante ha sido la experimental, cuantitativa y estadística (Siles, 2016). Como es lógico, la investigación está influenciada por el perfil profesional neopositivista tanto en la investigación básica como en la aplicada. Este tipo de enfoque (positivista), que es el preponderante desde el origen de los métodos y usos científicos, ha constreñido la enfermería al ámbito del método experimental y la tecnología alejándola de su auténtico epicentro epistemológico.

Se ha realizado una reflexión teórica sobre la naturaleza del cuidado como interacción entre profesional y paciente en el contexto de la estética de la enfermería (la ciencia que estudia las emociones y sentimientos que se derivan de esa interacción) (Siles \& Solano, 2016). Se llevó a cabo un análisis comparativo de los principios de dos paradigmas: el racional tecnológico y el sociocrítico valorando el papel que en cada uno de ellos se le confiere tanto al cuidador (enfermera) como al cuidado (persona en situación de cuidados o paciente). Desde los presupuestos del paradigma racional tecnológico donde la comunicación no ocupa un lugar central, el rol del enfermero es de mero dispensador de técnicas de forma estandarizada y el paciente se erige en un consumidor pasivo de prescripciones y aplicaciones terapéuticas. Estos papeles cambian sensiblemente desde los esquemas del paradigma Habermasiano donde la comunicación se muestra como una herramienta de cambio en la práctica clínica y en cuyo proceso el profesional de los cuidados utiliza la comunicación de forma científica tanto para comprender la realidad del paciente como para implicarlo de forma activa-participativa en la planificación de sus situaciones de salud-enfermedad (Siles, 2016).

\subsection{La Enfermería basada en la narrativa}

La Enfermería basada en la narrativa (EBN) tiene en cuenta los procesos de construcción social de la experiencia que se derivan de las vivencias y puede servir de puente entre la objetividad científica y la subjetividad experimentada (Siles \& Solano 2017; Siles \& Solano 2014; Siles, 2014). En este sentido textos como los de Marta Allué (1996, 1997); Sampedro (1996); Comas (2009) y Díaz (2008) pueden servir para comprender los sentimientos vividos frente al dolor y la enfermedad.

La poesía de los cuidados constituye un complemento de la EBN y es una fuente de conocimientos para enfermería (Holmes \& Gregory, 1998). Watson (2008) afirma que a través de la poesía de los cuidados se puede encontrar la verdad transcendente del acto enfermero. Birx (1994) indica que a través de este instrumento se puede analizar la trascendencia de los encuentros y las interacciones especialmente intensos entre personas. la poesía de los cuidados. En el campo de la medicina la aplicación de la literatura tiene una larga trayectoria (Greenhalgh, 1999; Kleinman, 1989). En este sentido, autores como Baños (2003) y Barbado (2007) proponen el estudio de diferentes obras literarias durante la formación médica, entre ellas cabe mencionar las obras (citadas por Baños, 2003 y Barbados, 2007) de Chéjov (1860-1904), Conan Doyle (1859-1930), William Somerset (1874-1965), o John Keats (1795-1819), destacado como el padre de la poesía médica. En España, son conocidas las obras (citadas por Baños, 2003 y Barbados, 2007) de Gregorio Marañón (1887-1960) o Pedro Laín Entralgo (1908- 2001) entre otros.

La introducción de la literatura en el currículo del ámbito de las Ciencias de la Salud no es reciente, diferentes facultades de Estados Unidos y otros países han implantado asignaturas de literatura y poesía desde la década de los años 70 (Baños, 2003). 


\subsection{La sociopoética}

Con la finalidad de generar una construcción colectiva del conocimiento nace la sociopoética, que trata de establecer una dialéctica entre el mundo interno y personal (conocimientos, creencias, valores...) y el mundo externo (instituciones, laboral, familiar, social, normas, leyes...), permitiendo objetivar el mundo que le rodea (Huerta-Rosas, 2008). La sociopoética es un método de investigación que se ocupa del estudio de las formas de expresión que a través de los sentidos y la experiencia provocan en las personas situaciones de intersubjetividad en procesos de enseñanza aprendizaje, salud, relaciones sociales... (Gauthier, 1999; Siles, 2014). El carácter intersubjetivo de la sociopoética se ha puesto de manifiesto en algunos estudios (Chocarro, 2013; Cody, 1995; Santos, 2005). Diferentes autores afirman que constituye un puente entre la ciencia y el arte potenciando la creatividad, la intuición y la sensibilidad de las personas (Paiva, Batista, \& Carneiro, 2003).

A continuación se presenta el proyecto detallado, con el objetivo general de desarrollar una herramienta de reflexión que contribuya a la gestión emocional y de los sentimientos de los alumnos de enfermería en sus prácticas clínicas. Asimismo, se ha pretendido alcanzar los siguientes objetivos específicos:

- Identificar las emociones y los sentimientos inspirados por poemas relacionados con la práctica clínica.

- Valorar la incidencia del proceso de enfermería en la construcción social de los sentimientos (sociopoética).

\section{El proyecto}

Con la finalidad de que los estudiantes tengan una mirada más humanista ante los diferentes procesos de vida-salud-enfermedad, en la Facultad de Ciencias de la Salud de la Universidad de Alicante, el grupo de investigación Enfermería y Cultura de los Cuidados lleva más de una década trabajando con metodologías docentes innovadoras que permiten un acercamiento hacia la subjetividad no sólo de la persona a la que están tratando sino también una indagación personal ante los diferentes fenómenos y eventos de la realidad que viven (Siles \& Solano, 2019).

\subsection{Fases del proceso}

El proceso se llevó a cabo en 3 fases (figura 1).

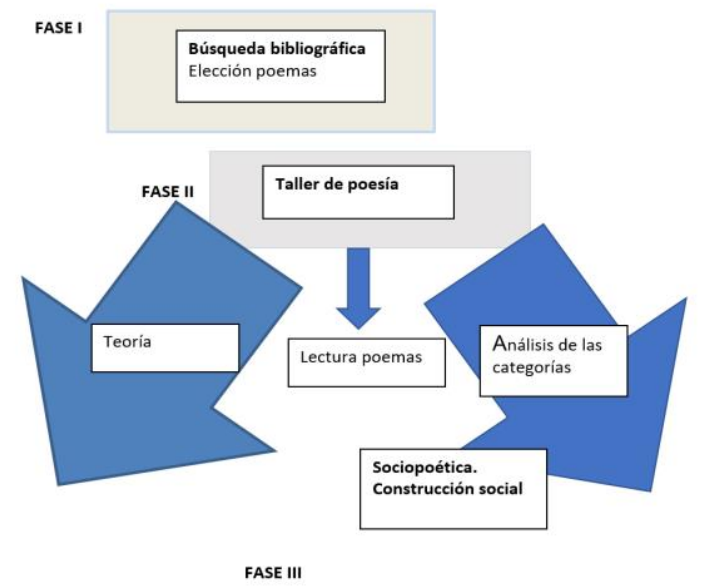

Fig. 1. Fases del proceso 


\subsection{Fase I. Búsqueda bibliográfica}

Determinación del problema de estudio, cuestiones de investigación y objetivos.Para determinar la unidad de observación, análisis y la muestra, se han seguido las orientaciones de Martínez-Salgado $(2012$, p.616) “(...) la lógica que orienta este tipo de muestreo - y lo que determina su potencia - reside en lograr que los casos elegidos proporcionen la mayor riqueza de información posible para estudiar en profundidad la pregunta de investigación." De forma que la muestra objeto de estudio ha estado compuesta por el total de los alumnos que cursan la asignatura optativa Enfermería y cultura de los cuidados 2015-16 $(n=46)$. El criterio de inclusión queda definido por ser alumnos que cursan esta asignatura y por haber experimentado contacto previamente con la práctica clínica y por tanto tener experiencias con diferentes tipos de pacientes (bien como alumnos en prácticas o como profesionales). El número de la muestra ha venido determinado por el principio de saturación teórica.

\subsection{Fase III. Taller de poesía.}

Se llevó a cabo en 2 sesiones.

Primera sesión: se realizó una introducción acerca de los conocimientos teóricos de las diferentes técnicas que se abordan, como son: Enfermería basada en la narrativa, poesía de los cuidados y la sociopoética. Solicitud de participación voluntaria en el estudio (30 minutos).

Segunda sesión: se establece la lectura de poemas definidos previamente para tal fin. Para ello se realiza primero una lectura, reflexión y respuesta por escrito de manera subjetiva e individualizada a las cuestiones planteadas (ver cuestiones a reflexionar ${ }^{\star}$ ) (1 hora y 30 minutos).

A continuación se plasma un ejemplo práctico de poesía (ver en anexos otras poesías utilizadas en el taller de poesía).

Adela llamando a su madre en el aire (Siles, 2019, p.104).

Aquella noche de luna ausente

el eco de su voz resonaba

a lo largo del oscuro pasillo

ensombreciendo aún más

la antiséptica atmósfera

de aquel hospital

donde el destino dentro de un sobre lacrado

estaba escrito prescribiendo

que la única ceremonia posible

era la despedida final.

Aquella noche de invocación incesante

toda su fuerza suplicante

se concentraba en un mismo fin

repitiendo una y otra vez

la misma palabra sin desmayo

llamándola como cuando era niña

anhelante del consuelo y la tierna calidez

de la mujer que la había traído al mundo

...su madre

la madre que volvía a lomos del aire.

Aquella noche de llanto silente

la mano de Adela rasgaba el aire de la habitación

ansiando hallar el último asidero

y buscando el encuentro de la otra mano

para que guiara su postrera travesía

mientras repetía una y otra vez

mamá, mamá, mamá

Aquella noche de luna ausente, invocaciones incesantes

y llantos silentes 


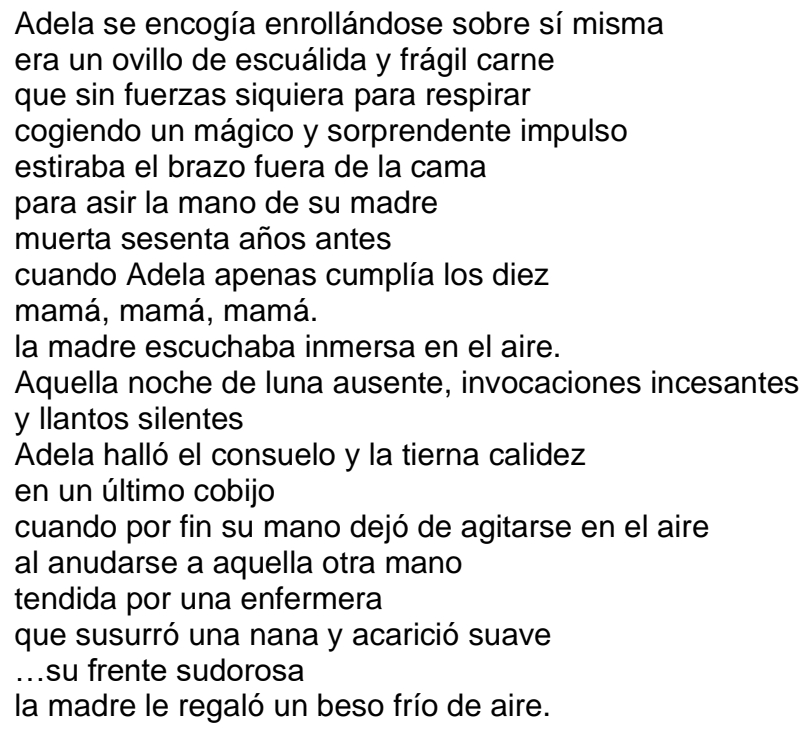

Posterior a la lectura del poema, se deberá realizar un análisis personal e individual que invite a la reflexión sobre las siguientes cuestiones*:

1. ¿Qué describe el poema?

2. ¿Qué sentimientos te inspira?

3. ¿Qué papel juega la enfermería en estas situaciones?

4. ¿Tiene algo que ver la humanización de los cuidados en este contexto?

5. ¿Qué relación puede existir entre el poema y el cuidado centrado en las ersonas?

6. ¿Qué utilidad le ves a la comunicación como técnica de trabajo en este poema?

Posteriormente, en grupos de 5 personas se procederá a debatir acerca de las emociones y sentimientos que les despierta la lectura del poema. El alumno recuerda a la luz de los poemas situaciones similares que ha vivido en sus prácticas clínicas y realiza un esfuerzo para expresar los sentimientos derivados de la misma; es decir vincula el sentimiento con la situación que lo inspira mediante una construcción narrativa. Para expresar los sentimientos rememorados, el alumno reflexiona y desarrolla una narrativa que acaba confiriendo un significado tanto a las vivencias como a los sentimientos.

Para garantizar las cuestiones éticas, se les proporcionó un documento informando acerca de las características del proyecto, su finalidad, así como su forma de difusión requiriéndoles su firma si estaban de acuerdo en participar.

\subsection{Fase III. Sociopoética}

El papel de los sentimientos en la construcción emocional es indisociable del entorno. Las prácticas clínicas constituyen una situación que influye en la generación y modulación de los sentimientos y, paralelamente, en la construcción personal. Se retoman los pequeños grupos establecidos a la fase II, así como la categorización de sentimientos propuesta por Heller (2004).

Posteriormente se realiza un análisis colectivo de todos los participantes. El resultado es la concienciación de los sentimientos y cierta objetivación (sociopoética) de los mismos al ser expuestos y valorados de forma intersubjetiva o colectiva (Fleuri, Prefácio, Santos, Gauthier, Figueiredo, \& Petit, 2008). Con la finalidad de ayudar al alumnado en la clasificación y categorización de emociones primarias y secundarias y sentimientos, los investigadores elaboraron un cuestionario, de acuerdo con las categorías y subcategorías propuestas por Heller (2004) (ver Figura 2, abajo) Este cuestionario se pasó a los alumnos tras explicarles las características propias de cada una de esas categorías y solicitarles que identificaran aquellas que consideraran como parte de su experiencia clínica de acuerdo con la vivencia experimentada retrospectivamente ya que su periodo de práctica finalizó hace 8 meses ( 2 horas). 


\section{Análisis de datos producidos en los talleres}

Con el fin de organizar la información, guiar la comprensión y la interpretación y hacer visible su recuperación y socialización, los investigadores tomaron el criterio de realizar el análisis temático del contenido. La información recibida en el taller de poesía fue transcrita literalmente respetando la subjetividad de los participantes y el reconocimiento del espacio temporo espacial. Se extrajeron citas textuales que dieron lugar a la generación de categorías y códigos.

Para el taller de sociopoética se utilizó la categorización de sentimientos propuesta por Heller (2004) (Ejemplo detallado A. Fig.2)

A. Sentimientos estéticos:

A.1 Lo bello: sentimientos de conciliación con el ser humano y el entorno.

A.2 Lo sublime: trascendencia, empatía significativa en momentos especialmente cruciales: nacimiento, muerte, etc.

A.3 Melancolía: sentimientos inspirados en situaciones que provocan recuerdos acerca de pérdidas, rememoraciones de experiencias vividas (nostalgia, añoranza).

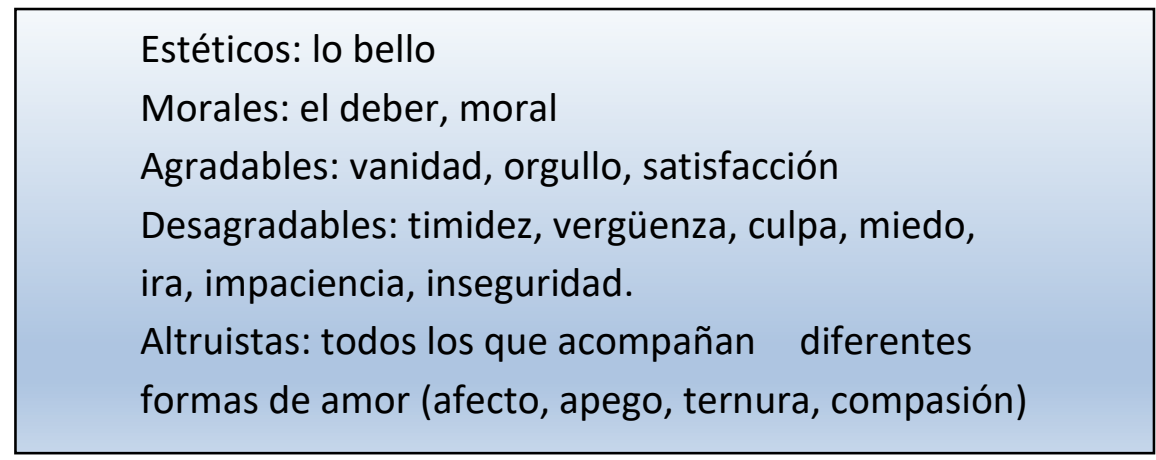

Fig. 2. Sentimientos categorizados (adaptados de Heller, 2004).

Fuente: Cuestionario de emociones-sentimientos vividos durante el trabajo clínico de enfermería. Elaboración propia.

La utilización de dos fuentes diferentes de información (por un lado, la poesía con la lectura individualizada de poemas y por otro lado el compartir las sensaciones y emociones con el grupo a través de la sociopoética) ayudó en el proceso de triangulación de los datos. Por otro lado, el empleo de una teoría como es la propuesta por Heller, proporcionó consistencia a los resultados obtenidos.

Los principales resultados ponen de manifiesto que las emociones primarias relativas a satisfacción son las que adquieren un mayor porcentaje en los alumnos, seguidas de las de emociones de tristeza, en concreto la subcategoría de pena. Con respecto a las emociones secundarias el mayor porcentaje otorgado corresponde a las integradas dentro del concepto amor, seguidas del concepto sorpresa.

Los sentimientos vinculados con la categoría de desagradables como son la ira, la impotencia, inseguridad son los que han alcanzado un mayor porcentaje entre los participantes. En definitiva, las emociones y sentimientos positivos potencian la actividad del cuidado y la atención hacia la persona, por otro lado cuando son negativos se genera un distanciamiento de la situación. 


\section{Guias para estudios siguientes}

En futuras investigaciones se recomienda dedicar un mayor tiempo a explicaciones teóricas sobre las características propias de las emociones y sentimientos evidenciando ejemplos que ayuden a identificarlas.

La elección de textos (narrativos o poesía) debe ir vinculada con una temática concreta, por ejemplo: el dolor, la muerte, el cáncer... ello facilitará el proceso de identificación y categorización de las emociones.

Con la finalidad de que no se produzca un sesgo por el recuerdo experimentado a lo largo del tiempo y afloren emociones y sentimientos con facilidad, se sugiere la realización de este tipo de talleres coetáneos en el tiempo a la realización de la actividad práctica asistencial.

La principal limitación de este proyecto ha sido la identificación de las emociones, especialmente las primarias, ya que han tenido una dificultad especial para ser detectadas por los alumnos, dado su carácter espontáneo sin tiempo a la reflexión y la rapidez de la respuesta conductual (aumento ritmo cardíaco, sudoración, nervios, entre otros). En definitiva la propuesta planteada se trata de una guía para futuros estudios ya que este tipo de intervenciones debe de contemplar las características propias de los participantes, así como las peculiaridades del propio entorno y contexto donde se desarrolla dicha intervención.

\section{Aplicabilidad en otros contextos}

La literatura en sí, tanto la narrativa como la poesía constituye un gran recurso docente para enseñar determinados aspectos a futuros profesionales como son los estudiantes de medicina, enfermería, trabajo social, psicología... que difícilmente pueden ser estudiados y aprendidos en las diferentes asignaturas (básicas y obligatorias) propias de su curricula.

La utilización de materiales narrativos en los procesos de enseñanza aprendizaje favorece tanto a los estudiantes como a los profesionales que interaccionan con personas a adquirir un conjunto de habilidades comunicativas, desarrollar sensibilidad y ciertas actitudes de escucha activa frente a diversas situaciones complejas planteadas por los procesos vidasalud y enfermedad.

La aplicabilidad de este tipo de herramientas que en definitiva dotan de voz a los personas que viven y padecen enfermedades puede ayudará a los profesionales y estudiantes en la comprensión de fenómenos complejos experimentados en la vida de las personas mejorando así la aplicación de su práctica profesional.

\section{Conclusiones}

A través de la lectura de los diferentes poemas escogidos se han podido evidenciar las emociones y sentimientos que afloran al recordar diferentes momentos de la práctica asistencial aplicando el proceso de atención de enfermería en diferentes tipos de pacientes (López, 2000). Los alumnos experimentan sentimientos y emociones tanto positivos como negativos, aunque los positivos prevalecen sobre los negativos mediante el desarrollo de un proceso de adaptación (López, 2000).

Una de las aportaciones más significativas de este proyecto la constituye la revelación de la poesía como herramienta pertinente para clarificar la naturaleza transcendente de la enfermería al constatar un hecho tan obvio como ignorado que es su objeto sujeto que es el ser humano (Birx, 1994; Siles \& Solano, 2017; Siles, 2018b). La persona como núcleo principal (su materia prima), con la que la enfermera trabaja para producir su obra. Una materia prima frágil, delicada, compleja, individual e irrepetible de ahí la importancia de su carácter transcendente (Siles \& Solano, 2016). 
La sociopoética permite vincular la parte del sentimiento que surge del interior filtrado a través de las creencias, valores, conocimientos y actitudes o "habitus" del alumno, con su correspondencia exterior o campo social; es decir, con la incidencia de la cultura institucional propia del escenario donde se desarrolla la acción (Huertas Rosas,2008; Gauthier, 1999; Pavia et al., 2003).

\section{Referencias}

Allué, M. (1996). Perder la piel. Barcelona: Seix Barral.

Allué, M. (1997). Sobrevivir en la UCI. Enfermería Intensiva, 6, 29-34

Baños, J.E. (2003). El valor de la literatura en la formación de los estudiantes de medicina. Educación Médica, 6(2), 37-43. Recuperado en 14 de abril de 2020, de http://scielo.isciii.es/scielo.php?script=sci_arttext\&pid=S1575$18132003000200005 \&$ Ing=es\&tlng=es

Barbado,FJ. (2007). Medicina y Literatura en la formación del médico residente. Anales de Medicina Interna, 24(4), 195-200.

Birx, E. (1994). The poetry of nursing. Clinical Nurse Spectrum, 8, 292-293.

Borges, J. L. (1969). El elogio de la sombra. Ed. Emecé. Buenos Aires.

Cody, W. (1995). Intersubjectivity: Nursing's contribution to the explication of its postmodern meaning. Nursing Science Quarterly, 8(2), 52-54.

Comas, J. (2009). Crónicas del linfoma. Madrid: Rey Lear.

Chocarro, L. (2013). La intersubjetividad y su relevancia en los cuidados de Enfermería. Index Enferm, 22(4), 219-221. doi: http://dx.doi.org/10.4321/S1132-12962013000300006

Darwin, C. (1998). La expresión de las emociones en los hombres y los animales. Madrid: Alianza

Díaz, J. L. (2008). El sufrimiento medicalizado. Cultura de los cuidados, 12(23), 50-56.

Fleuri, R., Prefácio, I. N., Santos, I., Gauthier, J., Figueiredo, N.M.A., \& Petit, S. H. (2004). Práctica de investigación en Ciencias Humanas y Sociales - Abordaje Sociopoético. Rio de Janeiro: Atheneu, 2.

Gauthier, J. (1999). Sociopoética- Encontro entre arte, ciência e democracia na pesquisa em ciências humana e social, enfermagem e educação. Rio de Janeiro. Anna Nery-UFRJ.

Greenhalgh, T. (1999). Narrative based medicine: Narrative based medicine in an evidence based world. BMJ Clinical Research, 318(7179), 323-325. https://doi.org/10.1136/bmj.318.7179.323

Heller, A. (2004). Teoría de los sentimientos. Ediciones Coyoacán, México.

Holmes, V., \& Gregory, D. (1998). Writing poetry: a way of knowing nursing. Journal Advance Nursing 8(6), 1191-4.

Huerta-Rosas, A. (2008). La construcción social de los sentimientos desde Pierre Bordieu Iberoforum. Revista de Ciencias Sociales de la Universidad Iberoamericana, III(5), 1-11. [Consultado: 10 de Abril de 2020]. Disponible en: https://www.redalyc.org/articulo.oa?id=2110/211015579005

Kleinman, A. (1989). The Illness Narratives: Suffering, Healing, And The Human Condition. New York: Basic Books.

López, S.R. (2000). Implicación emocional en la práctica de la enfermería. Cultura de los Cuidados, $4(7 / 8), 172-180$.

Martínez-Salgado, C. (2012). El muestreo en investigación cualitativa. Principios básicos y algunas controversias. Ciência \& Saúde Coletiva, 17(3), 613-619.

Millán, G. (2007). Veneno de escorpión azul. Diario de vida y de muerte. Santiago de Chile: Ediciones Universidad Diego Portales.

Paiva, L.M., Batista, V.A., \& Carneiro, L. (2003) Sociopoética e enfermagem: una aproximación en la investigación con adolescentes. Revista Baiana de Enfermagem, 18, 89-96. 
Sampedro, R. (1996). Cartas desde el infierno. Barcelona: Planeta.

Santos, I. (2005). Sociopoética: un puente para "cuidar - investigar" en enfermería. Index de Enfermería, 14(50), 35-37. Recuperado en 09 de octubre de 2020, de http://scielo.isciii.es/scielo.php?script=sci_arttext\&pid=S1132$12962005000200007 \&$ Ing=es\&tlng=es

Siles, J. (2014). El humanismo en enfermería a través de la sociopoética y la antropoética. Cultura de los Cuidados (Edición digital), 18(38), 9-13. doi.org/10.7184/cuid.2014.38.01>

Siles, J. (2016). La utilidad práctica de la Epistemología en la clarificación de la pertinencia teórica y metodológica en la disciplina enfermera. Index de Enfermería, 25(1-2), 86-92. Recuperado de http://scielo.isciii.es/scielo.php?script=sci arttext\&pid=S1132$12962016000100020 \& \operatorname{lng}=e s \& n r m=i s o \& t \operatorname{lng}=e s$

Siles, J. (2018a). Educación Superior e Investigación Aplicada: sinergias y conveniencias [video]. Rev Paraninfo Digital, XII (28). Recuperado de http://www.index-f.com/para/n28/v005.php.

Siles, J. (2018b). La humanización del cuidado a través de las narrativas y la poesía como producto de la investigación aplicada. Cultura de los Cuidados, 22(52), 9-15. Recuperado de http://dx.doi.org/10.14198/cuid.2018.52.01

Siles, J. (2019). La estructura del aire. Madrid: Verbum.

Siles, J., \& Solano, C. (2014). Qualitative Research and Narrative Sources in the Context of Critical and Renal Cares. Open Journal of Nursing, 4(7), 385-401 doi: 10.4236/ojn.2014.46045

Siles, J., \& Solano, C. (2016). Sublimity and beauty: A view from nursing aesthetics. Nursing Ethics, 23(2), 154-166.

Siles, J., \& Solano, C. (2017). Poesía y cuidados: un instrumento para la gestión de emociones y sentimientos en enfermería. Enfermería: Cuidados Humanizados, 6(2), 2393-6606. doi.org/10.22235/ech.v6i2.1463

Siles, J., \& Solano, C. (2019). Pensamiento crítico, autotevaluación y estética en la práctica clínica de enfermería. Barcelona. Octaedro.

Vigotsky, L. (2004). Teoría de las emociones. Akal, Madrid.

Watson, J. (2008). Nursing: The Philosophy and Science of Caring. Colorado: University Press of Colorado.

\section{ANEXOS}

Turbación Ilegítima (José Siles) Reflexión sobre la pena, el dolor, la desesperanza, la falta de sentido de la vida

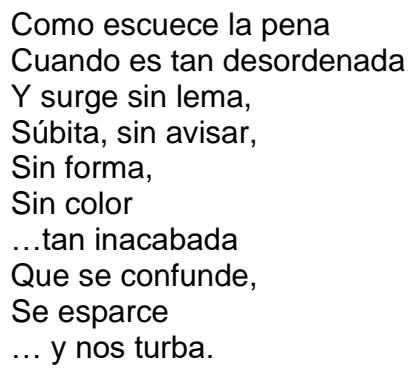

Veneno de escorpión azul Millán, «Fragmentos» (2009)

Diario morir / Diario vivir

Diario de vida / Diario de muerte

Hechos consumados / Deshechos consumados

El día a día. Células grandes.

En el umbral de la muerte / Cerca del fin

Poemas a la muerte / Poemas de despedida de la vida

Adiós al pasado

Testamento / Preparación para el viaje.

(...) El cáncer es el destructor de la ignorancia de mi cuerpo

se apropia de las riendas de mi respiración, 
auriga que conduce el carro de carne con jeringas,

desbocado hacia el despeñadero.

Somos una mayoría del cuerpo condenada a la muerte por una facción rebelde, suicida.

Células sin brújula me estrangulan.

Elogio de la sombra (Jorge Luis Borges) Reflexión sobre la ancianidad, las sociedades envejecidas y sus consecuencias a nivel individual y global. 2018

La vejez (tal es el nombre que los otros le dan)

puede ser el tiempo de nuestra dicha.

El animal ha muerto o casi ha muerto.

Quedan el hombre y su alma.

Vivo entre formas luminosas y vagas

que no son aún la tiniebla.

Buenos Aires,

que antes se desgarraba en arrabales

hacia la llanura incesante,

ha vuelto a ser la Recoleta, el Retiro,

las borrosas calles del Once

y las precarias casas viejas

que aún llamamos el Sur.

Siempre en mi vida fueron demasiadas las cosas;

Demócrito de Abdera se arrancó los ojos para pensar;

el tiempo ha sido mi Demócrito.

Esta penumbra es lenta y no duele;

fluye por un manso declive

y se parece a la eternidad.

Mis amigos no tienen cara,

las mujeres son lo que fueron hace ya tantos años,

las esquinas pueden ser otras,

no hay letras en las páginas de los libros.

Todo esto debería atemorizarme,

pero es una dulzura, un regreso.

De las generaciones de los textos que hay en la tierra

sólo habré leído unos pocos,

los que sigo leyendo en la memoria,

leyendo y transformando.

Del Sur, del Este, del Oeste, del Norte,

convergen los caminos que me han traído

a mi secreto centro.

Esos caminos fueron ecos y pasos,

mujeres, hombres, agonías, resurrecciones,

días y noches,

entresueños y sueños,

cada ínfimo instante del ayer

y de los ayeres del mundo

la firme espada del danés y la luna del persa,

los actos de los muertos,

el compartido amor, las palabras,

Emerson y la nieve y tantas cosas.

Ahora puedo olvidarlas. Llego a mi centro,

a mi álgebra y mi clave,

a mi espejo.

Pronto sabré quién soy.

\section{Notas biográficas:}

José Siles González. Escritor, doctor en Historia, pedagogo, enfermero y catedrático de la universidad de Alicante. Dirige la revista Cultura de los Cuidados. Asimismo coordina el grupo de investigación Enfermería y Cultura de los Cuidados y el grupo de Antropología Educativa de los Cuidados. Entre sus publicaciones destaca diferentes libros de poesía y narrativa en el ámbito de la literatura. Desde el punto de vista académico ha llevado a cabo diferentes proyectos de investigación en calidad de investigador principal y de colaborador, derivándose de ellos numerosos artículos de impacto internacional. 
MCarmen Solano Ruiz. Profesora Titular del Departamento de Enfermería de la Universidad de Alicante, doctora y licenciada en Antropología Social y Cultural. Diplomada en Enfermería. Su línea de trabajo giran en torno a la investigación cualitativa aplicada a las prácticas clínicas, antropología social y educativa. Ha colaborado en diferentes proyectos de investigación, derivándose de ellos trabajos que han sido premiados por su calidad científica y publicados en revistas con un alto factor de impacto. 